Peter Niesen

\title{
Internationale Politische Theorie: Eine disziplinengeschichtliche Einordnung
}

Es hat sich eingebürgert, mit »Internationaler Politischer Theorie» (IPT) die normative Theorie internationaler Politik zu bezeichnen. Versteht sie sich als »Internationale Ethik«, also als präskriptive Theorie für das staatsübergreifende Handeln von Zwangs- und Gewaltakteuren, kann IPT nicht umhin, die Nachfolge des klassischen Völkernaturrechts anzutreten, wie sich an der Rückkehr von Theorien des gerechten Krieges ablesen lässt. Aber auch als Theorie legitimer trans-, supra- und internationaler Institutionen kann IPT nicht automatisch die Anmaßung präskriptiver naturrechtlicher Aussagen umgehen. Während die am Einzelstaat orientierte Politische Theorie ein naturrechtliches Selbstverständnis schließlich durch eine konsequent demokratiewissenschaftliche Einbettung und Orientierung am öffentlichen Vernunftgebrauch vermeiden konnte, ist im Blick auf die globale Ebene noch nicht absehbar, wie eine analoge Dezentrierung der Autorität Politischer Theorie gelingen könnte.

Der Sache nach ist Internationale Politische Theorie in der deutschsprachigen Politikwissenschaft, in der Politischen Theorie ebenso wie den Internationalen Beziehungen (IB) kein unbeschriebenes Blatt. Dass es dennoch mehr als dreißig Jahre gedauert hat, bis der Ausdruck im deutschsprachigen Kontext eine Rolle spielen und in einer Frankfurter Konferenz aus verschiedenen Perspektiven untersucht werden konnte $^{1}$, deutet an, dass keine Klarheit über Sinn, Skopus, Methoden und Aufgaben eines so bezeichneten Forschungsgebiets besteht. Viel weniger noch herrscht Konsens darüber, ob sich hinter dem Ausdruck eine neue politikwissenschaftliche Teildisziplin verbirgt.

Offensichtlich ist allein, dass es sich beim Etikett Internationale Politische Theorie (IPT) um eine Rückübersetzung aus dem Englischen handelt. In der englischsprachigen Forschung hat sich der Ausdruck für ein klar umgrenztes Gebiet eingebürgert, das aber diskussions- und erweiterungsbedürftig erscheint. Grob gesagt ist dort International Political Theory die normative Theorie der internationalen Beziehungen. Damit ist häufig eine dezidiert philosophische Zugangsweise verbunden. Die jüngst gegründete Standing Group on International Political Theory des European Consortium for Political Research (ECPR) formuliert:

»International political theory (IPT) [...] applies a philosophical mode of enquiry to international politics. IPT brings together political philosophers whose interests are wholly or predominantly in international affairs with scholars of international politics or the EU

$1 \mathrm{Zu}$ der von den Sektionen für Internationale Politik und Politische Theorie/Ideengeschichte vom 10.-12.6.2010 veranstalteten Konferenz vgl. die Tagungsberichte in der Zeitschrift für Politische Theorie (10/2010), in der Zeitschrift für Außen- und Sicherheitspolitik (im Erscheinen) und auf der Seite http://www.theorieblog.de. 
whose interests are philosophical or ethical in character« (ECPR Standing Group IPT 2010).

Dieselben Interessen charakterisieren die neue Zeitschrift International Political Theory der Edinburgh University Press, während die Webplattform internationalpolitical-theory.net einen fachlich etwas breiteren Rahmen absteckt und Themen internationaler Politik, des Völkerrechts und internationaler politischer Ökonomie unter dem Schirm der IPT zusammenfasst. Gemeinsam ist all diesen Initiativen, dass IPT als Teilmenge der Politischen Theorie oder Politischen Philosophie gesehen wird. Sie enthalte sany work of political theory or philosophy which reflects on issues that transcend the horizon of a single nation-state « (IPT-net 2010). Die Internationalen Beziehungen im angelsächsischen Sprachraum teilen diese Charakterisierung, ohne dass sie damit die IPT aus dem Skopus der IB hinausfallen sähen. Mit entwaffnender Selbstsicherheit versammelt der Band »Fifty Key Thinkers in International Relations « die Vertreter der Forschungsrichtung IPT (immerhin John Rawls, Charles Beitz, David Held, Terry Nardin und Michael Walzer) unter dem Titel »International Political Theory/International Ethics« (Griffiths et al. 2009). Mit der Gleichsetzung ist zugleich ein Problem bezeichnet: vorausgesetzt, IPT umfasst die normative Theorie der internationalen Politik, ist dann Internationale Politische Theorie dasselbe wie Internationale Ethik? Führt der normative Zugang der IPT dazu, dass das gesamte Feld einer »Moraltheorie internationaler Beziehungen « (Müller 2004: 130) inkludiert wird? Um diese Fragen zu beantworten, muss zunächst kurz an die Begriffsgeschichte von International Political Theory erinnert werden (Abschnitt 1), bevor dann das Problem aus disziplinengeschichtlicher Perspektive angegangen werden kann (Abschnitt 2). Die These dieses Beitrags ist, dass Internationale Politische Theorie, die sich allein als Internationale Ethik, also als präskriptive Theorie für das Handeln von Zwangsund Gewaltakteuren versteht, nicht umhin kann, die Nachfolge des klassischen Völkernaturrechts anzutreten und so dessen problematische Erblast einstreichen muss.

\section{Internationale Politische Theorie in Internationalen Beziehungen und Politischer Theorie}

Unkontrovers dürfte sein, dass das Untersuchungsgebiet der IPT das Gebiet grenzüberschreitender Politik ist. Der Ausdruck selbst, um dies gleich eingangs anzusprechen, scheint etwas unglücklich gewählt, weil heutige Ansätze ganz überwiegend das Internationale in seiner strengen Bedeutung nur mehr als einen unter mehreren Gegenstandsbereichen untersuchen. Dies entspricht der gewandelten Realität, auf die die Disziplinen reagieren. Wohin man auch schaut, treten im traditionellen Bereich des zwischenstaatlichen Raumes neben den Staaten weitere Akteure auf, werden politische Entscheidungen in überstaatlichen governance-Arrangements getroffen, spielen schließlich kosmopolitische Bürgerengagements eine zunehmende Rolle, die alle in einem konventionellen Verständnis internationaler Beziehungen als Interaktion von Staaten ohne Relevanz wären. Aber es lässt sich bezweifeln, dass der Sache mit einer alternativen Bezeichnung wie »globale politische Theorie« wirklich gedient 
wäre. Nicht alle Fragen der IPT haben einen universalen Skopus. Auch Beiträge zur Theorie regionaler Integration, die das enge Verständnis des internationalen als eines zwischenstaatlichen Bereichs längst hinter sich gelassen haben (Neyer/Wiener 2010), verorten sich innerhalb der Internationalen Politischen Theorie. Viele, aber längst nicht alle wichtigen Forschungsfragen, die das Gebiet »jenseits des Staates « betreffen, richten sich gleich auf die globale Ebene. Daher bietet sich an, den Ausdruck Internationale Politische Theorie in einem umfassenden Verständnis weiter zu gebrauchen.

Die Begriffsgeschichte des Ausdrucks International Political Theory ist noch nicht geschrieben, aber in groben Zügen würde sie dualistisch ablaufen, unterschiedlich für die Internationalen Beziehungen und die Politische Theorie. Für die Internationalen Beziehungen ließe sie sich, grob zusammengefasst, folgendermaßen schildern. In den neunziger Jahren des vergangenen Jahrhunderts, inmitten konkurrierender Neuordnungsprozesse der Weltpolitik nach dem Ende des Kalten Krieges, können gleich zwei anti-realistische Makro-Trends Fuß fassen, die sich für die Teildisziplin autonomiesteigernd auswirken. Das ist erstens die zurückkehrende Gesellschaftstheorie, die die stone age metaphysics (Bertrand Russell) der Staatenanarchie und die empiristische Erkenntnistheorie zugunsten einer konstruktivistischen Ontologie und Epistemologie hinter sich lässt. Zweitens hat sich die politische Theorie in das bisherige normative Vakuum jenseits des Staates hineingekämpft und wird sich dort behaupten. In der Integration dieser beiden Trends gelingt den Internationalen Beziehungen im englischsprachigen Bereich eine stärkere Ausdifferenzierung und Behauptung ihrer Selbständigkeit gegenüber einer weiterhin positivistisch dominierten Politikwissenschaft; der politischen Theorie sichert sie gleichzeitig bleibende Anschlussfähigkeit.

Für den deutschsprachigen Bereich wird man sagen können, dass der Impuls zur Modernisierung der Internationalen Beziehungen weniger von der normativen politischen Theorie und stärker von der Gesellschafts- und Handlungstheorie, von der Orientierung an der International Society, an Lebenswelt und System, an der funktionalen Differenzierung der Weltgesellschaft, den Grenzen rationalistischer Erklärungsansätze usw. ausging. Darin ähnelt die deutschsprachige Diskussion der älteren »International Theory « (Wight 1960). Eine Orientierung an normativen Fragen ist in den Grundbegriffen zu identifizieren, bleibt aber implizit und wird nicht innerhalb der Teildisziplin ausformuliert, zumindest nicht in Auseinandersetzung mit der existierenden normativen politischen Theorie. Auch dort, wo Ansätze aus der politischen Theorie aufgegriffen wurden, wie etwa die Diskurstheorie von Jürgen Habermas (1981; 1992), wird deren Bedeutung in erster Linie in ihrem Beitrag als explanatorische Theorie gesehen und zunächst weniger in ihrem Anspruch als Theorie legitimer normativer Ordnungen. ${ }^{2}$ Im englischsprachigen Bereich geht umgekehrt die normative Theorie in Führung, auch wenn die Protagonisten natürlich auf die English School und andere gesellschaftstheoretische Ansätze zurückgreifen können. Aber die Gewichtung normativer Fragen ist diesseits und jenseits des Ärmelkanals eine andere. In den deutschsprachigen IB hat sich ein normativer Paradigmenwechsel, für den

2 Siehe im Anschluss an die ZIB-Debatte, Deitelhoff (2006), Niesen/Herborth (2007) sowie Kessler (2010, in diesem Heft). 
Forschertypen wie Chris Brown oder Terry Nardin stehen, nicht vollzogen. ${ }^{3}$ Für die Politische Theorie im deutschsprachigen Bereich ist das zumindest aus strategischer Sicht kein nachteiliger Zustand, denn er dokumentiert, dass die Internationalen Beziehungen nicht oder zumindest noch nicht in der Lage sind, ihre eigene Reflexionswissenschaft aus eigenen Beständen zu reproduzieren, sondern dass sie zumindest auf dem Gebiet der Theorie politischer Normativität auf die externe Expertise angewiesen sind. Dort, wo die politikwissenschaftliche Großforschung oder wichtige universitäre Fachbereiche von den Internationalen Beziehungen dominiert werden, werden politisch-theoretische Anteile also bisher auf eigenes Risiko reduziert.

Die Politische Theorie hat andere Schwierigkeiten, will sie sich als IPT neu entwerfen. Innerhalb der Politischen Theorie ist IPT bisher keine methodische Neuorientierung, sondern eine Neubestimmung des Skopus unter hergebrachten Methoden. Das rührt einerseits daher, dass die IPT in der Politischen Theorie, deren Begriffsgeschichte ungefähr zehn Jahre früher beginnt als in den Internationalen Beziehungen, als Fortführung eines bestehenden Paradigmas ansetzen konnte. 1979 hatte sich Charles Beitz in »Political Theory and International Relations « von der älteren »International Theory « abgegrenzt und erst einmal den Skeptizismus darüber widerlegen müssen, dass eine normative Theorie der internationalen Beziehungen wissenschaftlich respektabel sein kann (Beitz 1979). Beitz kann sich auf das Anfang der siebziger Jahre etablierte Arsenal des Rawls-Paradigmas stützen und die Ideen von Urzustand und Prinzipienkonstruktion, von Grundstruktur, wohlgeordneter Gesellschaft und Systemen fairer Kooperation versuchsweise auf den Globus ausdehnen. Beitz' Buch ist auch in einer anderen Hinsicht vorbildlich geblieben, nämlich in seinem Rückgriff auf die Geschichte des politischen Denkens in systematischer Absicht, die heute ganz wesentlich für die Produktivität der IPT innerhalb der Politischen Theorie ebenso wie innerhalb der Internationalen Beziehungen verantwortlich ist. ${ }^{4}$

Beitz' Meilenstein blieb innerhalb der Politischen Theorie lange ein Solitär und das Unternehmen einer systematischen IPT bis Anfang der neunziger Jahre des 20. Jahrhunderts marginal. Das Gros der Disziplin reagierte unvorbereitet auf die offenere weltpolitische Konstellation, die sich überschlagenden Prozesse der Globalisierung und die zunehmende Herausbildung von governance-Strukturen. Aber die RawlsMethode und, vielleicht ebenso wichtig, der Stil und die Fragestellungen von Robert Nozick: Welche Rechte haben wir? Was dürfen wir einander keinesfalls antun? Wann ist Gewaltanwendung moralisch akzeptabel? (Nozick 1974; vgl. auch Wolff 2011), die ja selbst in der Auseinandersetzung mit Rawls entwickelt wurden, stellten sich als für die kommende Neuorientierung geeignet heraus und sorgten für eine klare und kohärente Agenda. Man war in der Lage, sozusagen im eigenen Idiom, auf die neuen Herausforderungen globaler Politik zu reagieren, Forschungsfragen zu definieren und

3 Vgl. Nardin (1983), Brown (2002). In der Leistungsschau der »Neuen Internationalen Beziehungen« (2003) von Hellmann et al., die als Identitätsbrevier der Teildisziplin keineswegs überholt erscheint, ist die Neuausrichtung entlang normativer Themen mit Händen zu greifen, wird aber in keinem Beitrag ausdrücklich bearbeitet.

4 Ein sich jüngst vollziehender historical turn hat vor allem die normative Dimension der Klassiker der politischen Ideengeschichte betont. Siehe Brown et al. (2002); Jahn (2007). 
Fortschritte von Rückschritten zu unterscheiden. Einer der wichtigsten frühen Versuche, Internationale Politische Theorie auf dem von Beitz vorgegebenen Niveau zu betreiben, heißt nicht zufällig »Realizing Rawls« (Pogge 1989: 211-280). ${ }^{5}$ Dies erklärt, warum in der englischsprachigen International Political Theory die Arbeitsweisen und thematischen Festlegungen über die Jahre erstaunlich stabil geblieben sind, in der Kombination von prinzipienorientierter Theoriebildung mit konkreten Einzelfragen, in der Heranziehung präzise beschriebener, manchmal absurd anmutender Beispiele und Gedankenexperimente aus alltäglicher Interaktion, schließlich in der Komplementarität von idealer und nicht-idealer Theorie.

\section{Internationale Politische Theorie als Neo-Naturrecht?}

Dennoch hat sich durch den Rückgriff vor allem auf den Nozick-Stil, also den unmittelbaren moralphilosophischen Zugriff auf normative Einzelfragen, ein Problemdruck aufgebaut. Die Irritation über die behauptete Synonymie von International Political Theory und International Ethics hat einen Grund in der Sache, der an den heute zentralen Arbeitsfeldern der Internationalen Politischen Theorie, der Theorie des gerechten Krieges (einschließlich der Theorie humanitärer Intervention) sowie der Theorie globaler distributiver Gerechtigkeit, deutlich wird. Nardin (2006: 544-547) zufolge sind diese Arbeitsgebiete nicht zufällig die zentralen Betätigungsfelder der IPT geworden. Paradigmatisch sind solche Themen, die Implikationen für die gerechtfertigte globale Ausübung von Zwang und Gewalt haben. IPT forscht ja nicht nur über zwangsbewehrte Normen, etwa indem sie Rechenschaft ablegt über die möglichen Quellen von Normativität, die Logik der Argumentation für die eine oder andere Position oder die Bedeutung der verwendeten Begriffe, sondern macht auch selbst normative Aussagen, die, wie es auf dem Gebiet des Politischen nicht anders sein kann, gerechtfertigte Zwangsausübung betreffen. Nardin ist ebenfalls in der Diagnose zu folgen, dass sich Aussagen zu grenzüberschreitendem Zwang und grenzüberschreitender Gewalt in der neueren Literatur konkretisiert und radikalisiert haben. Cecile Fabre (2011) etwa kombiniert Fragen globaler Gerechtigkeit und des gerechten Krieges, wenn sie in einem Text zu »Krieg und Subsistenz« dafür argumentiert, dass unter bestimmten Bedingungen die Ärmsten der Welt ein Recht haben, gegen die Begüterten in anderen Ländern in den Krieg zu ziehen, falls die Letzteren nicht Abstand davon nehmen, sich an der Verursachung sehr schwerwiegender Armut zu beteiligen. Fabres Auffassung dient hier nicht dazu, eine inhaltliche Position zu illustrieren; sie dokumentiert vielmehr ein bestimmtes Verständnis der Aufgabe, die IPT heute haben soll: Die Aufgabe der Theoretikerin wird in der Formulierung präskriptiver Aussagen vermutet; ihre Arbeit kreist um die Rechtfertigung von Gewaltanwendung, und ihre Aussagen betreffen nicht etwa die institutionellen Systeme, innerhalb derer eine prozedural nachvollziehbare Antwort auf normative Fragen gege-

5 Ein früher Blick über paradigmatische Grenzen hinweg findet sich im Schlusskapitel von Young (1990). 
ben werden kann, sondern die Gründe, die Handlungen wie den Krieg der Besitzlosen gegen die Besitzenden auf direktem Wege legitimieren oder delegitimieren. Auch die in jüngster Zeit neu erblühende Diskussion um das ius in bello ist kaum mit der Reform des Völkerrechts verknüpft, sondern konzentriert sich auf die direkte Autorisierung moralisch gebotenen Handelns (McMahan 2009).

Die erste Frage der Internationalen Politischen Theorie ist hier nicht: Wie soll eine legitime Ordnung jenseits des Staates aussehen? Vielmehr lautet sie: Welche Normen sollen und dürfen gewaltsam durchgesetzt werden? Gefragt wird nicht nach der Form globaler Institutionen, sondern nach den Inhalten präskriptiver Normen, die innerhalb oder außerhalb solcher Institutionen, und notfalls auch gegen sie gelten und die Akteure zu bestimmten Handlungsweisen autorisieren. IPT in ihrem Selbstverständnis als präskriptive Theorie gewalt- oder zwangsbewehrten Handelns jenseits des Staates, also als internationale Ethik, tritt damit inhaltlich und disziplinär an die Stelle des klassischen Völkernaturrechts. Unter »Naturrecht« ist dabei nicht eine bestimmte, kosmologisch oder theologisch festgelegte Konzeption, sondern einfach der Inbegriff von universell zu erzwingenden Normen zu verstehen, Normen, auf die beliebige Akteure überall zu Recht zurückgreifen können, wenn sie sich überlegen, wie sie interagieren sollen (Nardin 2009). Das Problem der Produktion von Naturrecht ist das Problem der wissenschaftlichen Produktion universell zu erzwingender Normen.

Aus historischer wie systematischer Perspektive steht Internationale Politische Theorie als Nachfolgedisziplin in der Tradition des Völkernaturrechts. Wenn Rawls, Held, Walzer, Beitz und Nardin als ihre charakteristischen Vertreter gelten können, so stehen neben Immanuel Kant auch stets Hugo Grotius, Samuel von Pufendorf, Emmerich de Vattel und andere Autoren in der Ahnenreihe, Kant zufolge »lauter leidige Tröster«, deren Bemühung um präskriptive Aussagen in der internationalen Politik »nicht die mindeste gesetzliche Kraft hat, oder auch nur haben kann« (Kant 1795: 355). Mit der Polemik Kants gegen Grotius, Pufendorf und Vattel wird ins theoretische Völkerrecht eine Unterscheidung zwischen zwei Strategien eingeführt: die »leidigen Tröster« verstehen ihre Aufgabe so, dass von ihnen eine klare Kasuistik präskriptiver Aussagen für das Handeln im unübersichtlichen weltweiten Naturzustand zwischen den Völkern und Individuen erwartet wird, während Kant alle Anstrengungen in die Modernisierung des Vernunftrechts, in seine positive Verrechtlichung und völkerrechtliche Institutionalisierung legt. Wird IPT als Internationale Ethik verstanden, liegt die Entscheidung für das Vattel-Paradigma auf der Hand: IPT sorgt dann für die ethische Bewertung von Handlungen und die unmittelbare Handlungsorientierung von Akteuren. Wird die IPT in der Nachfolge Kants verstanden, als Theorie legitimer internationaler Ordnungen, liegt ihre Aufgabe in der Orientierung von Begründung und Reform von Institutionen, die den einzelnen Akteuren den unmittelbar handlungsleitenden Zugriff auf ethische Gründe in Konfliktsituationen entziehen müssen. Ein zentrales Problem der Internationalen Politischen Theorie liegt mithin heute darin, an welchen Typ von Aufgabenbeschreibung sie anknüpfen will: Soll sie als Internationale Ethik Rechtfertigungen für zwingendes und gewaltsames Handeln produzieren oder sich vielmehr auf legitime institutionelle Ordnungen konzentrieren? 
Aber auch wenn eine Entscheidung gegen IPT als Internationale Ethik und zugunsten der Kantischen Variante einer normativen Theorie globaler Verrechtlichung fällt, ist der Problemdruck, der aus den Ursprüngen des Forschungsansatzes im klassischen Naturrecht resultiert, noch nicht grundsätzlich überwunden. Auch die Argumentation für neue grenzüberschreitende Institutionen, für die Neudefinition oder Erweiterung der Kompetenzen bestehender Institutionen, betrifft die Installation und Operationsweise von Zusammenhängen bindenden Entscheidens - in aller Regel auf der Basis von Zwang und Sanktionsdrohung. Ein zentrales Beispiel sind Aussagen über globale redistributive Gerechtigkeit, die sich typischerweise auf die Etablierung eines obligatorischen Besteuerungssystems und nicht auf individuelle Philanthropie oder Ethik richten (Nardin 2006: 455). Auch der Austritt aus dem globalen Naturzustand ist, darüber ist sich Kant völlig im Klaren, immer noch ein Zug innerhalb des Naturrechts, wenn zwingende Mechanismen der Koordination dort hergestellt werden, wo es vorher keine gab. Die Schwierigkeit, die präskriptive Ansätze in der IPT erzeugen, liegt daher heute weniger darin, dass die Beteiligten zu »leidigen Tröstern« würden, weil die von ihnen entwickelten Kodizes keine Gesetzeskraft erlangen können, wie Kant vermutete. Die Problematik ihrer Vorgehensweise liegt vielmehr darin, dass sie für ihre individuellen Aussagen wie für ihre institutionellen Designs Gesetzeskraft, also Nachachtung verschaffendes Zwangspotenzial in Anspruch nehmen in anderen Worten: dass sie für ihre Positionen nicht allein moralische Akzeptabilität, sondern politische Legitimität beanspruchen. Die Reflexion dieses Problems wird man der Internationalen Politischen Theorie ansinnen müssen.

Wie kann Internationale Politische Theorie auf produktive Weise auf ihre unvermeidliche naturrechtliche Erblast reagieren? Dazu lohnt es sich, einen Blick auf die Art und Weise zu werfen, in der die nicht-internationale Politische Theorie mit dem Problem umgegangen ist. In der Blütezeit der politischen Theorie legitimer staatlicher Ordnung, die bis Mitte der neunziger Jahre des vergangenen Jahrhunderts anhielt, hatte sich eine Lösung herauskristallisiert, wie sich trotz der Unvermeidlichkeit präskriptiver Aussagen die Produktion von Naturrecht durch die politische Theorie vermeiden ließ. Die dort gefundene Lösung kann der Einfachheit halber innerhalb des Rawls-Ansatzes skizziert werden, auch wenn sie dort erst auf die beharrliche Kritik von Habermas hin entwickelt wurde (Rawls 1998; Habermas 1992). Innerhalb des demokratischen Staates konnte normative politische Theorie dem naturrechtlichen Problemdruck durch zwei Entscheidungen entgehen, erstens indem sie ihr Augenmerk auf die Gestalt einer kontingenterweise bestehenden institutionellen Ordnung richtete, und zweitens indem sie ihre Argumente in letzter Instanz an den Souverän in Gestalt der Mitbürger adressierte, da nur der souveräne demos dazu in der Lage ist, sie über verfassungsförmig festgelegte Kanäle in legitimes positives Recht umzuwandeln (Rawls 1997; vgl. Niesen 2007). Die staatsbezogene politische Theorie siedelt ihre präskriptiven Aussagen also in zweifacher Hinsicht innerhalb einer bereits bestehenden normativen Ordnung an. Der Fokus auf die institutionelle Grundstruktur einer bereits bestehenden Gesellschaft führt dazu, dass die Rechtfertigung von Zwang ex nihilo nicht vorkommt: der Naturzustand ist innerhalb des Staates, so ließe sich der Punkt in der traditionellen naturrechtlichen Redeweise ausdrücken, immer schon 
überwunden. Fragen nach der Mobilisierung von Ressourcen und Zwangspotenzialen entstehen nur innerhalb eines bereits errichteten Institutionensystems. Den weiteren, genuin naturrechtlichen Schritt einer Rechtfertigung des Staats gegenüber einem hypothetischen vorstaatlichen Zustand brauchte sich die politische Theorie nicht mehr zuzumuten. Die Einbettung politischer Theorie innerhalb eines demokratischen Systems führt dann in einem zweiten Schritt dazu, dass philosophische Expertise sich dort in legitime Entscheidungen transformieren lässt, wo sie sich in der demokratischen Willensbildung bewährt. Es reicht nicht länger aus zu zeigen, dass die Anwendung von Zwang und Gewalt moralisch akzeptabel wäre: will sie auch politisch legitim sein, muss sie sich im öffentlichen Vernunftgebrauch durchsetzen. Diese Vermittlung von Argumenten der politischen Philosophie durch die Willensbildung der Bürger und der ihnen verantwortlichen Repräsentanten hat für die innerstaatliche politische Theorie die Problematik der Produktion naturrechtlicher Aussagen absorbiert. Insoweit für die legitime Transformation normativer Gründe in öffentliche Gewalt kein alternatives Verfahren zur Verfügung steht, muss politische Theorie immer auch Demokratiewissenschaft sein, das heißt ihre demokratischen Verwirklichungsbedingungen mitreflektieren. ${ }^{6}$

Dieser Königsweg ist der Internationalen Politischen Theorie versperrt, denn der institutionelle Bezugsrahmen für legitimes kollektiv verbindliches Entscheiden steht in ihr selbst zur Diskussion: die Einschätzung der entgegengesetzten Optionen von Weltrepublik und Staatenbund, von Weltstaat und Staatenwelt, von Global Governance oder völkerrechtlicher Globalverfassung gehören zu den grundlegenden Debatten der IPT. Auch wenn aus empirischen Gründen heute einiges dafür spricht, die Existenz eines globalen Zusammenhangs politischer Interaktion zu unterstellen und die Gegenposition (von der Rawls selbst noch fest überzeugt ist), bereits die Umrisse einer solchen Grundstruktur wären allererst zu etablieren, nur mehr als abstrakte Möglichkeit mitzuführen, so wäre dieser Annahme zufolge doch nur das eine der beiden post-naturrechtlichen Merkmale politischer Theorie, nämlich die Existenz eines institutionellen Rahmens, erfüllt. Die zentrale zweite Frage, was denn die demokratie-analogen Verwirklichungsbedingungen präskriptiver politisch-theoretischer Aussagen sein sollen, ist aber damit noch nicht vorentschieden, ja nicht einmal angesprochen; sie muss mit der institutionellen Struktur, auf die abgezielt wird, variieren. Weder liegt die zukünftige Gestalt der Weltordnung fest, so dass eine normative Orientierung künftige Institutionen universeller Erzwingbarkeit vorwegnehmen könnte, noch ist die Frage nach den relevanten Entscheidungsträgern und damit nach einem angemessenen Forum der Rechtfertigung, das die demokratische Auseinandersetzung im Kontext des Staates beerben würde, bereits entschieden. Es wäre wenig überzeugend, stipulativ nun einen kosmopolitischen demos an die Stelle des

6 Die hier angemahnte Reflexivität der Politischen Theorie verhält sich genau invers zur von Brunkhorst (2010, in diesem Heft) thematisierten Reflexivität der Luhmannschen Evolutionsund Weltgesellschaftstheorie. Sie betrifft nicht die Beteiligung der Theoretikerin an der gesellschaftlichen Sinnproduktion, die sie gleichzeitig beobachtet, sondern die Beteiligung des gesellschaftlichen Vernunftgebrauchs an den Geltungsbedingungen theoretisch identifizierter Normen. 
nationalstaatlichen zu setzen und als letzte Instanz des Appells für präskriptive Aussagen einfach festzulegen. Die Problemstellung des präsumtiven Adressaten lässt sich nicht einfach auf die kosmopolitische Ebene projizieren und darauf vertrauen, dass legitime globale Koordination die Koordination im demokratischen Staat im großen Maßstab spiegelt. Wenn nicht die Unausweichlichkeit einer Entwicklung demonstriert werden kann, an deren Ende sich kosmopolitische Demokratie als verbleibender globaler Kontext einer verfassungsmäßigen Verrechtlichung von Zwang und Gewalt herausstellen wird (Held 1995), kann nicht im Vorgriff auf alternative Rechtfertigungsebenen wie die einer bleibend intergouvernementalen globalen Ordnung verzichtet werden. In dieser Situation ist unklar, wie eine ihrer demokratischen Einbettung entsprechende Dezentrierung der Position der Theoretikerin auf globaler Ebene gelingen kann.

Dazu kommt, dass sich die Politische Theorie, deren Institutionenverständnis häufig von konzeptuellem Konservatismus geprägt ist, nicht länger leisten kann, sich in der sterilen Opposition zwischen den institutionellen Alternativen von staatenweltlichem Internationalismus und kosmopolitischer Republik, die die Dekade nach dem Kalten Krieg strukturierte, einzurichten, ohne auf die sozialwissenschaftlichen Beschreibungsangebote neuer institutioneller Optionen einzugehen. Ohne in den angrenzenden Disziplinen der Internationalen Beziehungen, der Soziologie und des Völkerrechts auf Diagnosen neuer Prozesse der Institutionalisierung zurückzugreifen (Wiener 2010, in diesem Heft; Forst 2010, in diesem Heft), ohne den Rekurs auf internationale Regime, governance-Strukturen oder Konstitutionalisierungsprozesse einer funktional differenzierten Weltgesellschaft, müsste Internationale Politische Theorie rückwärtsgewandt oder im schlechten Sinn utopisch werden. Allerdings kann sie ihrerseits den sozialwissenschaftlichen Fachkollegen den Einwand nicht ersparen, dass gerade eine institutionell pluralistische Entwicklung jenseits des Staates es nicht zulässt, stets und geradenwegs mit demselben Operator namens »demokratische Legitimität« auf die neuen Regulationsmodi zuzusteuern (Wiener 2010: 340). An spekulativen Entwürfen globalen legitimen Regierens fehlt es aufseiten der politischen Theorie nicht: Blaupausen für die gerechte Weltordnung bis in die Verästelungen ihrer Administration hinein finden sich allerorten. Was fehlt, sind Aussagen über die legitimen Wege, dorthin zu gelangen: eine Theorie des legitimen Institutionenwandels und eine Theorie des globalen öffentlichen Vernunftgebrauchs, auf deren Basis sich ein solcher Wandel kognitiv motivieren und bewerkstelligen ließe. Hier wären kooperative Forschungsfelder zwischen Internationaler Politischer Theorie und empirischer Sozialwissenschaft zu entwickeln, wo bisher nicht einmal gemeinsame Fragestellungen artikuliert werden. Wenn wir Wert darauf legen, die Unterscheidung zwischen Moralität und politischer Legitimität für Kontexte jenseits des Staates beizubehalten und weder die Komplexität der Realisierungsbedingungen institutioneller Ordnungen noch die Komplexität der Geltungsbedingungen Politischer Theorie zu unterschreiten, wäre die erste Frage der IPT, was einem methodischen Bekenntnis zur Demokratiewissenschaft auf internationaler Ebene entsprechen könnte. 


\section{Literatur}

Beitz, Charles 1979: Political Theory and International Relations, Princeton, NJ.

Brown, Chris 2002: Sovereignty, Rights and Justice: International Political Theory Today, Cambridge.

Brown, Chris/Nardin, Terry/Rengger, Nicholas (Hrsg.) 2002: International Relations in Political Thought: Texts in International Relations from the Ancient Greeks to the First World War, Cambridge.

Brunkhorst, Hauke 2010: Neustart - Kritische Theorie Internationaler Beziehungen, in: Zeitschrift für Internationale Beziehungen 17: 2, 293-315.

Deitelhoff, Nicole 2006: Überzeugung in der Politik. Grundzüge einer Diskurstheorie internationalen Regierens, Frankfurt a. M.

ECPR Standing Group IPT 2010: Standing Group on International Political Theory, in: http:// www.essex.ac.uk/ecpr/standinggroups/ipt/ipt.aspx; 19.9.2010.

Fabre, Cecile 2011: War and Subsistence, in: Pogge, Thomas (Hrsg.): Freedom from Poverty as a Human Right, Band 2, Oxford, i.E.

Forst, Rainer 2010: Was ist und was soll Internationale Politische Theorie?, in: Zeitschrift für Internationale Beziehungen 17: 2, 355-363.

Griffiths, Martin/Roach, Steven C./Solomon, M. Scott 2009: Fifty Key Thinkers in International Relations, 2. Auflage, London.

Habermas, Jürgen 1981: Theorie des kommunikativen Handelns, 2 Bände. Frankfurt a. M.

Habermas, Jürgen 1992: Faktizität und Geltung. Beiträge zur Diskurstheorie des Rechts und des demokratischen Rechtsstaats, Frankfurt a. M.

Held, David 1995: Democracy and the Global Order, London.

Hellmann, Gunther/Wolf, Klaus Dieter/Zürn, Michael (Hrsg.) 2003: Die neuen Internationalen Beziehungen, Baden-Baden.

IPT-net 2010: About the International Political Theory Website, in: http://international-politicaltheory.net/ipt-about.htm; 19.9.2010.

Jahn, Beate (Hrsg.) 2007: Classical Theory in International Relations, Cambridge.

Kant, Immanuel 1795: Zum ewigen Frieden. Ein philosophischer Entwurf, Band VIII, Akademie Ausgabe 1912, Berlin.

Kessler, Oliver 2010: Internationale Politische Theorie: Fehlentwicklung oder fehlende Entwicklung der IB?, in: Zeitschrift für Internationale Beziehungen 17: 2, 317-334.

McMahan, Jeff 2009: Killing in War, Oxford.

Müller, Harald 2004: Think Big! Der 11. September und seine Konsequenzen für die internationalen Beziehungen, in: Zeitschrift für Internationale Beziehungen 11: 1, 123-133.

Nardin, Terry 1983: Law, Morality, and the Relations of States, Princeton, NJ.

Nardin, Terry 2006: International Political Theory and the Question of Justice, in: International Affairs 82: 3, 449-465.

Nardin, Terry 2009: International Political Theory, in: Burchill, Scott/Devetak, Richard/ Donnelly, Jack/Linklater, Andrew/ Nardin, Terry/Patterson, Matthew/Reus-Smit, Christian/ True, Jacqui: Theories of International Relations, 4. Auflage, Houndmills, 284-310.

Neyer, Jürgen/Wiener, Antje (Hrsg.) 2010: Political Theory of the European Union, Oxford, i.E.

Niesen, Peter 2007: Politische Theorie als Demokratiewissenschaft, in: Buchstein, Hubertus (Hrsg.): Politische Theorie und Politikwissenschaft, Wiesbaden, 126-155.

Niesen, Peter/Herborth, Benjamin (Hrsg.) 2007: Anarchie der kommunikativen Freiheit. Jürgen Habermas und die Theorie der internationalen Politik, Frankfurt a. M.

Nozick, Robert 1974: Anarchy, State and Utopia, New York, NY.

Pogge, Thomas 1989: Realizing Rawls, Ithaca, NY.

Rawls, John 1997: Erwiderung auf Habermas, in: Philosophische Gesellschaft Bad Homburg/ Hinsch, Wilfried (Hrsg.): Zur Idee des politischen Liberalismus. John Rawls in der Diskussion, Frankfurt a. M., 196-262. 
Rawls, John 1998: Politischer Liberalismus, Frankfurt a. M.

Wiener, Antje 2010: Zur normativen Wende in den IB: Triangulation of a Different Kind, in: Zeitschrift für Internationale Beziehungen 17: 2, 335-354.

Wight, Martin 1960: Why is there no International Theory, in: International Relations 2: 1, 35-48.

Wolff, Jonathan 2011: The History of Analytic Political Philosophy, in: Beaney, Michael (Hrsg.):

The Oxford Handbook to the History of Analytic Philosophy, Oxford, i.E.

Young, Iris 1990: Justice and the Politics of Difference, Princeton, NJ. 